ARTICLE

Received 2 May 2016 | Accepted 29 Jul 2016 | Published 23 Sep $2016 \quad$ DOl: 10.1038/ncomms12783 OPEN

\title{
Subnatural-linewidth biphotons from a Doppler-broadened hot atomic vapour cell
}

Chi Shu ${ }^{1}$ Peng Chen ${ }^{1}$, Tsz Kiu Aaron Chow ${ }^{1}$, Lingbang Zhu ${ }^{1}$ Yanhong Xiao ${ }^{2}$, M.M.T. Loy ${ }^{1} \&$ Shengwang Du ${ }^{1}$

Entangled photon pairs, termed as biphotons, have been the benchmark tool for experimental quantum optics. The quantum-network protocols based on photon-atom interfaces have stimulated a great demand for single photons with bandwidth comparable to or narrower than the atomic natural linewidth. In the past decade, laser-cooled atoms have often been used for producing such biphotons, but the apparatus is too large and complicated for engineering. Here we report the generation of subnatural-linewidth $(<6 \mathrm{MHz})$ biphotons from a Doppler-broadened $(530 \mathrm{MHz})$ hot atomic vapour cell. We use on-resonance spontaneous four-wave mixing in a hot paraffin-coated ${ }^{87} \mathrm{Rb}$ vapour cell at $63^{\circ} \mathrm{C}$ to produce biphotons with controllable bandwidth (1.9-3.2 MHz) and coherence time (47-94 ns). Our backward phase-matching scheme with spatially separated optical pumping is the key to suppress uncorrelated photons from resonance fluorescence. The result may lead towards miniature narrowband biphoton sources.

\footnotetext{
${ }^{1}$ Department of Physics, The Hong Kong University of Science and Technology, Clear Water Bay, Kowloon, Hong Kong, China. ${ }^{2}$ Department of Physics, State Key Laboratory of Surface Physics, Key Laboratory of Micro and Nano Photonic Structures, Fudan University, Shanghai 200433, China. Correspondence and requests for materials should be addressed to S.D. (email: dusw@ust.hk).
} 
B iphotons (entangled photon pairs) are the benchmark tools in the field of quantum optics for probing fundamental quantum properties of light quanta such as the waveparticle duality and non-locality ${ }^{1}$. They have also played an important role in developing advanced technologies in quantum information processing ${ }^{2}$. The quantum-network protocols based on efficient photon-atom interaction require photons have bandwidth sufficiently narrower than the atomic natural linewidth ${ }^{3,4}$. Here we are interested in generating frequency-anticorrelated biphotons whose sum frequency is fixed and the biphoton bandwidth refers to the spectrum of individual photons. With a fast time-resolved detector, narrowband biphotons can be used to generate pure heralded single photons with the bandwidth equal to the biphoton bandwidth $^{5}$. In earlier days, spontaneous parametric down conversion using nonlinear crystals ${ }^{6,7}$ and four-wave mixing in optical fibres ${ }^{8}$ were standard methods for producing biphotons. However, these biphotons have typically very wide bandwidth $(>\mathrm{THz})$ and short coherence time $(<\mathrm{ps})$, which make them extremely difficult for implementing photonic quantum information processing in an atomic-memory-based quantum network ${ }^{9,10}$. To solve this problem, many researches have focused on narrowing down the paired photon bandwidth by putting the nonlinear crystal inside an optical cavity ${ }^{11-14}$.

Subnatural-linewidth biphotons with controllable waveforms have been produced from spontaneous four-wave mixing (SFWM) in cold atoms $(10-100 \mu \mathrm{K})$ assisted with electromagnetically induced transparency (EIT) ${ }^{15-19}$ or cavity ${ }^{20}$. However, cold-atom systems require expert knowledge in laser cooling and trapping. A cold-atom apparatus is not only expensive, but also large and complicated in its vacuum-optical-electronic-mechanical configuration. Moreover, operating cold atoms for producing paired photons requires a complex timing control with a low duty cycle ${ }^{21}$.

If a hot atomic vapour cell can be used as an alternative source to produce narrowband biphotons, the system size and operation can be markedly simplified and the cost will be significantly reduced. However, the use of hot atomic vapour cell for producing narrowband biphotons has not been as successful as those with cold atoms. In an early demonstration in 2005, Lukin et al. ${ }^{22}$ generated nonclassical correlated light pulses from a room temperature ${ }^{87} \mathrm{Rb}$ atomic vapour cell with writing-reading pulse operation, but these photons are not time-frequency entangled and the photon number in each pulse is barely below the two-photon threshold. In this work, we focus on paired photon generation with time-frequency entanglement in continuous-wave operation mode. There have been some attempts in generating biphotons from hot atomic vapour cells, but with coherence time not exceeding $20 \mathrm{~ns}$, corresponding to a bandwidth of $>50 \mathrm{MHz}$ that is much wider than the typical atomic natural linewidths ${ }^{23-25}$.

Here we demonstrate generating subnatural-linewidth biphotons using on-resonance SFWM in a hot ${ }^{87} \mathrm{Rb}$ vapour cell assisted with EIT. Different from the off-resonance double-Raman scheme ${ }^{26}$ and diamond energy-level scheme ${ }^{24,25}$, where the photon bandwidth $(\sim 500 \mathrm{MHz})$ is determined by the Doppler-broadening decoherence time $(\sim 2 \mathrm{~ns})$ of the excited atomic states, the EIT effect can significantly prolong the photon coherence time and narrow down the bandwidth ${ }^{27}$. However, when directly applying the EIT-assisted SFWM scheme to a hot vapour cell, there is a serious noise problem: uncorrelated photons generated from resonance Raman scattering of the strong coupling laser field overwhelm the entangled photon pairs. To overcome this problem, we coat the inner wall of the cell with paraffin to increase the atomic ground-state coherence time and apply an additional strong optical-pumping beam to suppress the on-resonance scattering of the coupling field. The opticalpumping beam is spatially separated from the SFWM volume and does not interfere with the biphoton generation. This noise reduction together with other optical filtering allows us observing biphotons with a high contrast ratio.

\section{Results}

Biphoton generation with optical pumping. We produce subnatural-linewidth biphotons from a paraffin-coated ${ }^{87} \mathrm{Rb}$ vapour cell at $63^{\circ} \mathrm{C}$, as illustrated in Fig. 1. The details of the experimental set-up are described in the Methods section. In presence of two counter-propagating pump $\left(\omega_{\mathrm{p}}\right)$ and coupling $\left(\omega_{c}\right)$ laser beams, backward and phase-matched Stokes $\left(\omega_{\mathrm{s}}\right)$ and anti-Stokes $\left(\omega_{\mathrm{as}}\right)$ photon pairs are spontaneously generated. After spatial and frequency filtering, these photons are detected by two single-photon counting modules $\left(\mathrm{SPCM}_{\mathrm{s}}\right.$ and $\left.\mathrm{SPCM}_{\mathrm{as}}\right)$. We find the major noise source of uncorrelated photons is the on-resonance Raman scattering of the coupling field following the transition $\left|5 S_{1 / 2}, F=2\right\rangle \rightarrow\left|5 P_{1 / 2}, F=1\right\rangle \rightarrow\left|5 S_{1 / 2}, F=1\right\rangle$. These photons have the same central frequency and polarization as the anti-Stokes photons, and cannot be filtered away by the polarization and frequency filters. To clean up the residual atoms in the level $\left|5 S_{1 / 2}, F=2\right\rangle$, we apply a strong optical-pumping beam $\left(\omega_{\mathrm{op}}\right)$ on the transition $\left|5 S_{1 / 2}, \quad F=2\right\rangle \rightarrow\left|5 P_{3 / 2}, \quad F=1\right\rangle$. In order not to interfere with the SFWM transitions, the optical-pumping beam is aligned parallel to the Stokes-antiStokes mode without spatial overlap. The atoms in the level $\left|5 S_{1 / 2}, \quad F=2\right\rangle$ are optically pumped to the ground level $\left.5 S_{1 / 2}, F=1\right\rangle$, and thus the Raman scattering on the anti-Stokes channel is suppressed, owing to the long ground-state coherence time because of the paraffin coating.

To confirm that the spatially separated optical-pumping beam reduces the on-resonance Raman scattering of the coupling laser beam, we perform a control experiment of biphoton generation with and without optical pumping. The powers of the pump and coupling laser beams are 6 and $27 \mathrm{~mW}$, respectively. We observe that, after switching on the optical-pumping beam $(32 \mathrm{~mW})$, the photon detection rate on the anti-Stokes channel drops from $12,000 \mathrm{~s}^{-1}$ to $3,600 \mathrm{~s}^{-1}$, while the photon-pair rate is nearly unaffected. The contrast ratio between the biphoton coincidence (signal) and the accidental coincidence (noise) can be characterized by the normalized two-photon correlation functions $g_{\mathrm{s}, \mathrm{as}}^{(2)}(\tau)$, which are plotted in Fig. 2. It clearly shows that the peak value $\left(\left[g_{\mathrm{s}, \mathrm{as}}^{(2)}\right]_{m}\right)$ of the normalized two-photon correlation or the biphoton-noise contrast ratio increases by a factor of $\sim 3$ when we switch on the optical-pumping beam due to the reduction of the accidental coincidence counts.

Subnatural-linewidth biphotons. Figure 3 shows the biphoton waveforms. We fix the pump laser power at $6 \mathrm{~mW}$ and vary the coupling laser power, which is 27,9 and $1 \mathrm{~mW}$ for Fig. $3 \mathrm{a}-\mathrm{c}$, respectively. As expected, the two-photon correlation time becomes longer as we reduce the coupling laser power for narrower EIT window. Shown in Fig. 3a-c, the biphoton waveforms are exponential decay. The 1/e correlation times are 47,60 and $94 \mathrm{~ns}$, for Fig. 3a-c, respectively, all exceeding the natural lifetime $26.5 \mathrm{~ns}$ of $\mathrm{Rb} 5 P$ excited states. The blue theoretical curves are obtained numerically by taking into account the Doppler effect (Supplementary Note 1) and agree well with the experiment. This agreement allows us to extract the biphoton temporal wave function and joint spectrum. The bandwidths of these biphotons are 3.2, 2.6 and $1.9 \mathrm{MHz}$, for Fig. 3a-c, respectively (Supplementary Fig. 1). They are substantially narrower than the natural linewidth of $6 \mathrm{MHz}$ of $\mathrm{Rb} \mathrm{D} 1 / \mathrm{D} 2$ lines.

To characterize the nonclassical property of the photon-pair source, we confirm its violation of the Cauchy-Schwarz inequality ${ }^{28}$. Normalizing the coincidence counts to the accidental 
a

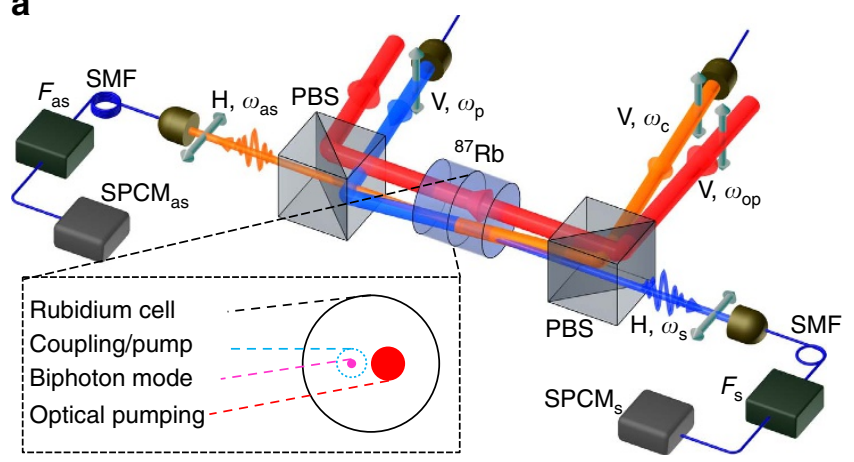

b

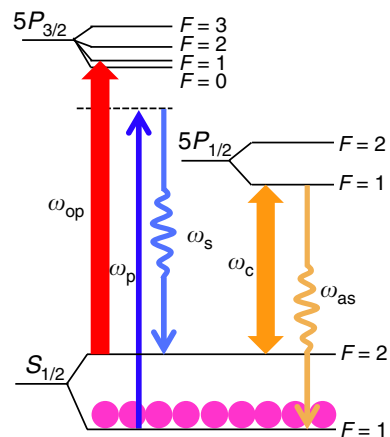

Figure 1 | Generating narrowband biphotons from a hot ${ }^{\mathbf{8 7}} \mathbf{R} \mathbf{b}$ vapour cell. (a) Experimental set-up. In the presence of counter-propagating vertically (V) polarized pump $\left(780 \mathrm{~nm}, \omega_{\mathrm{p}}\right)$ and coupling $\left(795 \mathrm{~nm}, \omega_{\mathrm{c}}\right)$ laser beams, horizontally polarized $(\mathrm{H})$ Stokes $\left(780 \mathrm{~nm}, \omega_{\mathrm{s}}\right)$ and anti-Stokes $\left(795 \mathrm{~nm}, \omega_{\text {as }}\right)$ photon pairs are spontaneously generated and coupled into two opposing single-mode fibres (SMF). Then, they pass through optical frequency filters $\left(F_{\mathrm{s}}\right.$ and $\left.F_{\mathrm{as}}\right)$, and are detected by two single-photon counting modules $\left(\mathrm{SPCM}_{\mathrm{s}}\right.$ and $\mathrm{SPCM}$ as $)$. The coincidence counts are recorded by a time-to-digit converter (Fast Comtec P7888). Two polarizing beam splitters (PBSs) are used as polarization filters to distinguish the paired photons from the two driving laser beams. The fibre-fibre coupling efficiency and SPCM detection efficiency are $80 \%$ and 50\%, respectively. Each optical frequency filter composes of a wide-band line filter and a narrowband etalon Fabry-Perot cavity filter. The etalon filters have free spectrum range $(F S R)=13.6 \mathrm{GHz}$. The bandwidth, transmission efficiency and the extinction ratio of the frequency filters are $350 \mathrm{MHz}, 80 \%$ and $60 \mathrm{~dB}$ for $F_{\mathrm{s}}$, and $80 \mathrm{MHz}, 30 \%$ and $40 \mathrm{~dB}$ for $F_{\mathrm{as}}$ respectively. In the inset, we make a zoom-in on the transverse cross-section of cell to clearly show the beam profiles of all incident lasers and the biphoton mode. The biphoton mode has a waist diameter $\left(1 / \mathrm{e}^{2}\right)$ of $250 \mu \mathrm{m}$ focused in the middle of the 0.5 -inch-long cell. The collimated coupling and pump laser beams are counter propagating and have the same $1 / e^{2}$ beam diameter of $1.4 \mathrm{~mm}$. The optical-pumping beam has a $1 / e^{2}$ diameter of $2 \mathrm{~mm}$ and does not overlap with the SFWM volume enclosed by the pump and coupling beams. The cell inner diameter is $10 \mathrm{~mm}$. (b) The relevant ${ }^{87} \mathrm{Rb}$ atomic energy-level diagram for backward SFWM and optical pumping. The strong optical-pumping laser is used to optically pump the atoms from the level $\left|5 S_{1 / 2}, F=2\right\rangle$ to $\left|5 S_{1 / 2}, F=1\right\rangle$, to suppress the on-resonance Raman scattering of the coupling beam.

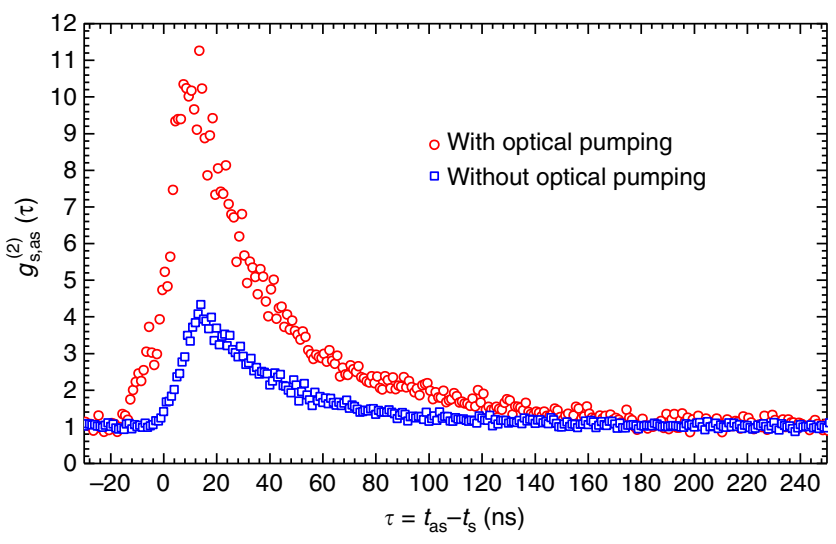

Figure 2 | Biphoton cross-correlation function with and without optical pumping. The control experiment is performed under $6 \mathrm{~mW}$ pump laser power and $27 \mathrm{~mW}$ coupling laser power. The red circles are measured in presence of the spatially separated optical-pumping beam ( $32 \mathrm{~mW})$ and the blue squares are measured without the optical-pumping beam.

background floor in Fig. $3 a-c$, we get the normalized crosscorrelation function $g_{s, a s}^{(2)}(\tau)$ with maximum values $\left[g_{s, a s}^{(2)}\right]_{m}=11 \pm 1$, $11 \pm 2$ and $6 \pm 1$. With the measured autocorrelations $g_{s, s}^{(2)}(0)=2.0 \pm 0.2$ and $g_{\mathrm{as}, \mathrm{as}}^{(2)}(0)=1.6 \pm 0.2$, we obtain violation of the Cauchy-Schwarz inequality $\left[g_{s, a s}^{(2)}(\tau)\right]^{2} /\left[g_{s, s}^{(2)}(0) g_{\mathrm{as}, \mathrm{as}}^{(2)}(0)\right] \leq 1$ by factors of $38 \pm 8,38 \pm 11$ and $11 \pm 3$, for Fig. 3a-c, respectively. We further verify the quantum nature of heralded anti-Stokes photons by measuring their conditional autocorrelation function $g_{c}^{(2)}$ (ref. 29). An ideal single-photon source gives $g_{c}^{(2)}=0$. A twophoton Fock state gives $g_{c}^{(2)}=0.5$, and a coherent state gives $g_{c}^{(2)}=1$. The measured $g_{c}^{(2)}$ as a function of coincidence window width $\Delta \tau$ are plotted as Fig. $3 \mathrm{~d}-\mathrm{f}$, which are below the two-photon threshold within their coherence time.
As we reduce the coupling laser power, the EIT bandwidth becomes narrower and the dispersion induced phase mismatching further constrains the biphoton joint spectrum ${ }^{27}$. Therefore, the biphoton bandwidth and coherence time are not determined by the lifetime of the excited states even though the photon pairs are indeed generated spontaneously. Figure $4 \mathrm{a}$ shows the measured decay time constant (square) and 1/e correlation time (circle) of the biphoton waveform as functions of coupling laser power. The longest correlation time at $1 \mathrm{~mW}$ coupling power approaches $\sim 100 \mathrm{~ns}$. On the other side, $\left[g_{s, a s}^{(2)}\right]_{m}$, the maximum value of the normalized cross-correlation function, decreases as we reduce the coupling power. Figure $4 \mathrm{~b}$ shows the $\left[g_{\mathrm{s}, \mathrm{as}}^{(2)}\right]_{m}$ and photon-pair generation rate as functions of the pump power. While the photon-pair rate is proportional to the pump laser power, the $\left[g_{s, a s}^{(2)}\right]_{m}$ drops at a high pump power. Limited by our available pump laser power of $7 \mathrm{~mW}$, we produced $\sim 2,000$ pairs per second. As the threshold of the $\left[g_{s, a s}^{(2)}\right]_{m}$ is 2.0 for violating the Cauchy-Schwarz inequality, the nonclassical property of the photon source is still preserved at $170 \mathrm{~mW}$ pump power, which corresponds to a generation rate of $\sim 47,000$ pairs per second.

\section{Discussion}

In summary, we demonstrate generation of subnatural-linewidth biphotons from a hot paraffin-coated ${ }^{87} \mathrm{Rb}$ vapour cell using EIT-assisted SFWM. The biphoton coherence time, controlled by the coupling laser power, can be as long as $94 \mathrm{~ns}$. The corresponding bandwidth of $1.9 \mathrm{MHz}$ is substantially narrower than the natural linewidth $6 \mathrm{MHz}$ of $\mathrm{Rb} \mathrm{D} 1 / \mathrm{D} 2$ transitions. It can be used to generate nearly pure heralded single photons ${ }^{5}$. The exponential waveform with tunable time constant is perfect for interacting with atoms ${ }^{30}$ and coupling to an optical cavity ${ }^{31}$. In this work, the heralding efficiency of the photon pairs is $3.1 \%$. This is determined by the small optical depth of $\sim 1$ of the atomic vapour cell, which is limited by the maximally allowed temperature $\left(<70^{\circ} \mathrm{C}\right)$ of the paraffin coating. If we can further increase the cell temperature while maintaining a low spin relaxation rate, we 


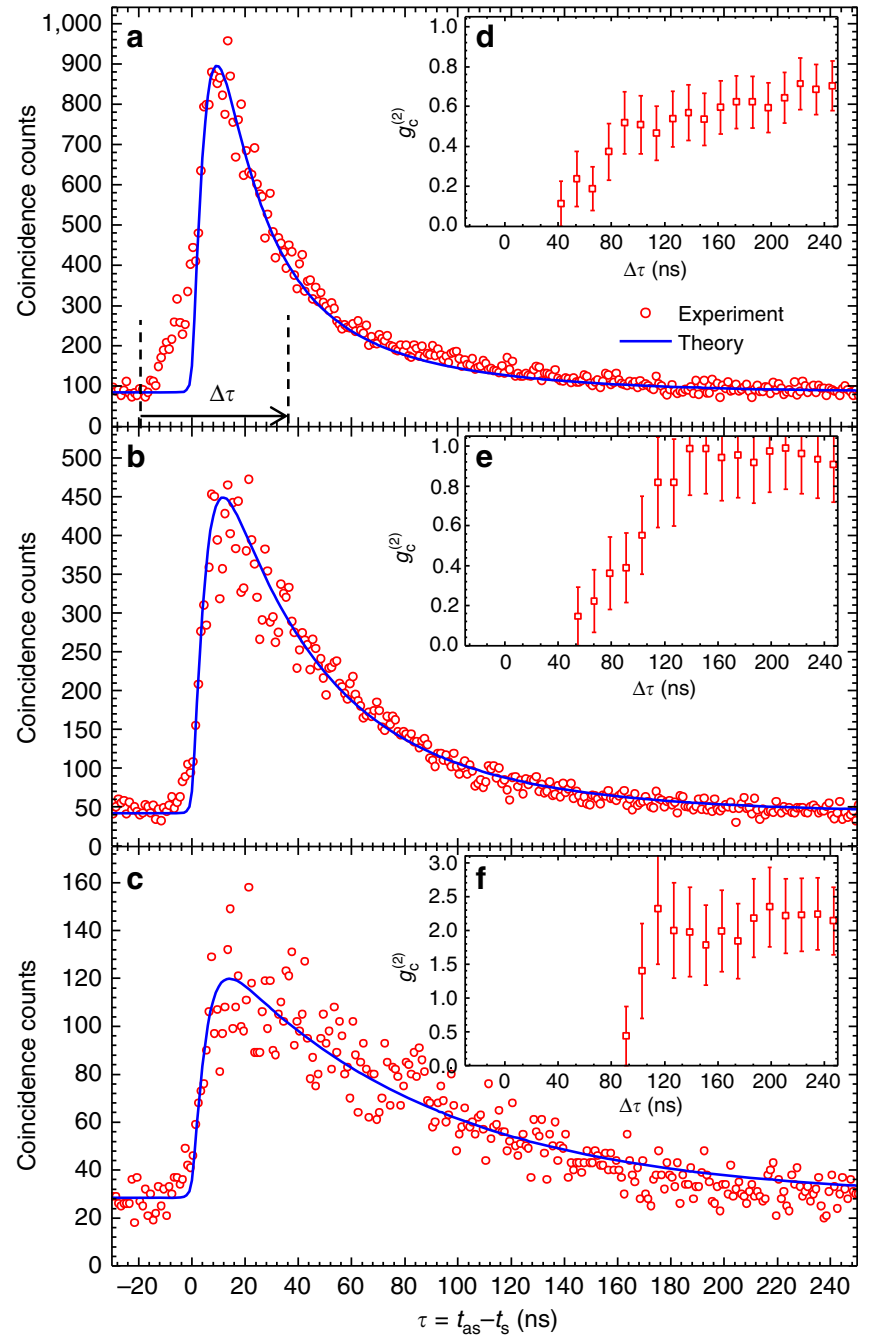

Figure 3 | Biphoton waveforms with controllable correlation time.

(a-c) Two-photon coincidence counts, collected over $600 \mathrm{~s}$ with $1 \mathrm{~ns}$ bin width, as a function of the relative time delay $\tau$ between paired Stokes and anti-Stokes photons. The incident pumping laser power is fixed at $6 \mathrm{~mW}$, while the incident coupling laser powers of $\mathbf{a}, \mathbf{b}$ and $\mathbf{c}$ are 27,9 and $1 \mathrm{~mW}$, respectively. The red circles are the experimental data. The solid blue curves are obtained numerically with the experimental parameters by taking into account the Doppler effect (Supplementary Note 1). The corresponding measured conditional autocorrelation function $g_{c}^{(2)}$ of heralded single anti-Stokes photons are plotted in $\mathbf{d}-\mathbf{f}$. The error bars are s.d.'s resulting from the statistical uncertainties of coincidence counts that can be reduced with longer data taking time.

expect to improve the heralding efficiency and generate biphotons with richer waveforms by engineering the spatial profile of the pump beam, as those demonstrated in cold atoms ${ }^{19}$.

The two key elements to make the SFWM narrowband biphoton generation feasible are the paraffin coating and the spatially separated optical pumping. The long ground-state coherence time preserved by the paraffin coating enables the efficient optical pumping, which is spatially separated from the biphoton generation volume, for the flying atoms without interfering the SFWM transitions. As a general state preparation method, the technology demonstrated here can be immediately applied to reduce incoherent photon noise thus improve the fidelity of the Raman-based quantum memory ${ }^{32}$. Future improvements could include improving the quality of optical frequency filtering (etalon
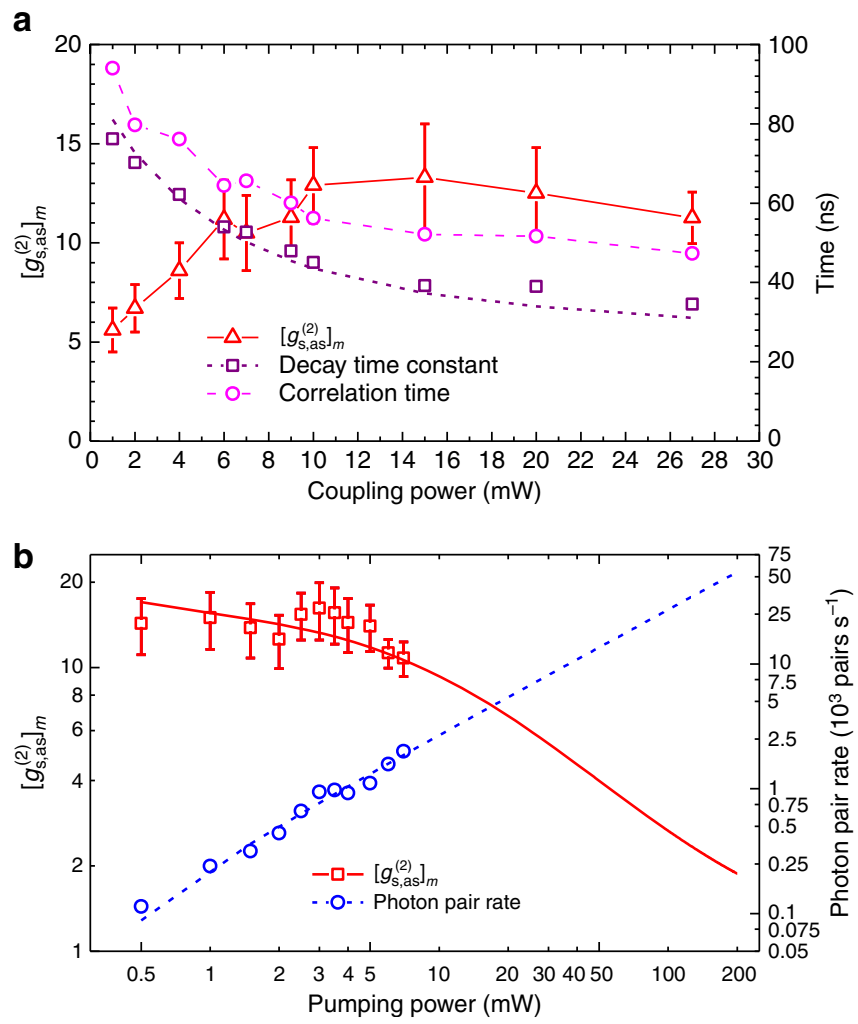

Figure 4 | Biphoton generation with controllable properties. (a) The biphoton properties versus the coupling laser power. The pump laser power is fixed at $6 \mathrm{~mW}$. The red triangular data represent the maximum of the normalized cross-correlation function, $\left[g_{\mathrm{s}, \mathrm{as}}^{(2)}\right]_{m}$. The decay time constant (purple, square) and 1/e correlation time (magenta, circular) are also plotted. The 1/e correlation time takes into account the rise time of the biphoton waveform in its rising edge. (b) $\left[g_{\mathrm{s}, \mathrm{as}}^{(2)}\right]_{m}$ and biphoton generation rate versus pump laser power. The fibre coupling efficiencies, filter transmissions and SPCM quantum efficiencies have been all taken into account to obtain the photon-pair generation rate. The coupling laser power is fixed at $27 \mathrm{~mW}$. The solid curve is a theoretical plot obtained by taking into account the uncorrelated noise photons (Supplementary Note 2). The error bars represent the s.d.'s resulting from statistical uncertainties of measured coincidence counts.

Fabry-Perot cavity, polarization filter, spatial-mode filter) and optimizing the power and spatial profile of the optical pump beam.

As compared with the cold-atom experiments ${ }^{33,34}$, the hot atomic vapour cell configuration is much simpler for operation and maintenance, and it is a continuous biphoton source. Our demonstration may lead to miniature narrowband biphoton sources based on atomic vapour cells for practical quantum applications and engineering.

\section{Methods}

Experimental set-up. The experimental set-up and associated atomic energy-level diagram are illustrated in Fig. 1. A paraffin-coated ${ }^{87} \mathrm{Rb}(99 \%$ enrichment purity, Precision Glassblowing Inc) vapour cell is placed in a temperature-stabilized hot-air heating oven, which is not shown in Fig. 1a, and is set at $63^{\circ} \mathrm{C}$ with fluctuation $<0.2^{\circ} \mathrm{C}$. The length of the vapour cell is $L=0.5 \mathrm{inch}$ and its inner diameter is $d=10 \mathrm{~mm}$. The longitudinal orientation of the cell is from east to west and there is no magnetic shielding in this experiment. The SFWM process is driven by two laser fields: the pump laser (D2 line: $780 \mathrm{~nm}, \omega_{\mathrm{p}}$ ) is locked to the ${ }^{85} \mathrm{Rb}$ transition $\left|5 S_{1 / 2}, F=2\right\rangle \rightarrow\left|5 P_{3 / 2}, F=3\right\rangle$, which is red detuned by $2.7 \mathrm{GHz}$ from the ${ }^{87} \mathrm{Rb}$ transition $\left|5 S_{1 / 2}, F=1\right\rangle \rightarrow\left|5 P_{3 / 2}, F=2\right\rangle$, and the coupling laser (D1 line: $795 \mathrm{~nm}, \omega_{\mathrm{c}}$ ) is on resonance to the transition $\left|5 S_{1 / 2}, F=2\right\rangle \rightarrow\left|5 P_{1 / 2}, F=1\right\rangle$. The vertically polarized pump and coupling laser beams are counter propagating with the same $1 / e^{2}$ beam diameter of $1.4 \mathrm{~mm}$. Backward, horizontally polarized, Stokes $\left(780 \mathrm{~nm}, \omega_{\mathrm{s}}\right)$ and anti-Stokes $\left(795 \mathrm{~nm}, \omega_{\mathrm{as}}\right)$ photon pairs are spontaneously 
generated, coupled into two opposing single-mode fibres, passing through optical frequency filters $\left(F_{\mathrm{s}}\right.$ and $F_{\mathrm{as}}$ ), and detected by two single-photon counting modules $\left(\mathrm{SPCM}_{\mathrm{s}}\right.$ and $\mathrm{SPCM}_{\mathrm{as}}$, Excelitas/PerkinElmer SPCM-AQRH-16-FC). The two-photon coincidence counts are recorded by a time-to-digit converter (Fast Comtec P7888) with a temporal bin width of $1 \mathrm{~ns}$. Two polarization beam splitters are used as polarization filters to distinguish the paired photons from the two driving laser beams. The spatially separated optical pumping is implemented by applying a strong vertically polarized optical-pumping beam $\left(\omega_{\mathrm{op}}\right)$ that is on resonance to the transition $\left|5 S_{1 / 2}, F=2\right\rangle \rightarrow\left|5 P_{3 / 2}, F=1\right\rangle$. The optical-pumping beam is aligned parallel to the pump-coupling beams without overlap. The laser beam profiles on the crosssection of the cell are shown in the inset of Fig. 1a. The optical-pumping beam, with a power of $32 \mathrm{~mW}$, has a $1 / e^{2}$ beam diameter of $2 \mathrm{~mm}$. The Stokes and anti-Stokes single-mode diameter on the cell centre is $250 \mu \mathrm{m}$. To further separate the generated photon pairs from the two driving laser beams, the pump and coupling laser beams are aligned with an angle of $\sim 0.5^{\circ}$ to the Stokes and anti-Stokes directions.

Normalized cross- and autocorrelation functions. The normalized two-photon cross-correlation function is defined as $g_{s, a s}^{(2)}(\tau)=\left\langle\hat{a}_{s}^{\dagger}\left(t_{\mathrm{s}}\right) \hat{a}_{\text {as }}^{\dagger}\left(t_{\mathrm{s}}+\tau\right) \hat{a}_{\mathrm{as}}\left(t_{\mathrm{s}}+\tau\right) \hat{a}_{\mathrm{s}}\left(t_{\mathrm{s}}\right)\right\rangle$ $/\left[\left\langle\hat{a}_{\mathrm{s}}^{\dagger} \hat{a}_{s}\right\rangle\left\langle\hat{a}_{\mathrm{as}}^{\dagger} \hat{a}_{\mathrm{as}}\right\rangle\right]$, where $\hat{a}_{\mathrm{s}, \mathrm{as}}^{\dagger}$ and $\hat{a}_{\mathrm{s} \text {,as }}$ are the creation and annihilation operators of the Stokes and anti-Stokes fields, respectively. The experimental $g_{s, a s}^{(2)}(\tau)$ is obtained by normalizing the two-photon coincidence counts to the flat background floor of accidental coincidence counts. The normalized autocorrelation functions are defined as $g_{\mathrm{s}, \mathrm{s}}^{(2)}(\tau)=\left\langle\hat{a}_{\mathrm{s}}^{\dagger}(t) \hat{a}_{\mathrm{s}}^{\dagger}(t+\tau) \hat{a}_{\mathrm{s}}(t+\tau) \hat{a}_{\mathrm{s}}(t)\right\rangle /\left[\left\langle\hat{a}_{\mathrm{s}}^{\dagger} \hat{a}_{\mathrm{s}}\right\rangle^{2}\right]$ and $g_{\mathrm{ass}, \mathrm{as}}^{(2)}(\tau)=\left\langle\hat{a}_{\mathrm{as}}^{\dagger}(t) \hat{a}_{\mathrm{as}}^{\dagger}(t+\tau) \hat{a}_{\mathrm{as}}(t+\tau) \hat{a}_{\mathrm{as}}(t)\right\rangle /\left[\left\langle\hat{a}_{\mathrm{as}}^{\dagger} \hat{a}_{\mathrm{as}}\right\rangle^{2}\right]$. The autocorrelation functions are measured using a fibre beam splitter.

Theoretical calculation of biphoton waveforms. The theoretical curves in Fig. 3 are obtained numerically following the Schrodinger picture approach ${ }^{27}$ by integrating over the Doppler-broadening profile (Supplementary Note 1). The solid theoretical curve in Fig. 4b is obtained by taking into account the uncorrelated noise photons (Supplementary Note 2).

Data availability. The data that support the findings of this study are available from the corresponding author on request.

\section{References}

1. Pan, J.-W. et al. Multiphoton entanglement and interferometry. Rev. Mod. Phys. 84, 777-838 (2012).

2. Bouwmeester, D., Ekert, A. K. \& Zeilinger, A. The Physics of Quantum Information (Springer-Verlag, 2000).

3. Kimble, H. J. The quantum internet. Nature 453, 1023-1030 (2008).

4. Duan, L.-M., Lukin, M. D., Cirac, J. I. \& Zoller, P. Long-distance quantum communication with atomic ensembles and linear optics. Nature 414, 413-418 (2001).

5. Du, S. Quantum-state purity of heralded single photons produced from frequency-anticorrelated biphotons. Phys. Rev. A 92, 043836 (2015).

6. Harris, S. E., Oshman, M. K. \& Byer, R. L. Observation of tunable optical parametric fluorescence. Phys. Rev. Lett. 18, 732-734 (1967).

7. Burnham, D. \& Weinberg, D. Observation of simultaneity in parametric production of optical photon pairs. Phys. Rev. Lett. 25, 84 (1970).

8. Fulconis, J., Alibart, O., Wadsworth, W. J., Russell, P. S. J. \& Rarity, J. G. High brightness single mode source of correlated photon pairs using a photonic crystal fiber. Opt. Express 13, 7572-7582 (2005).

9. Simon, C. et al. Quantum repeaters with photon pair sources and multimode memories. Phys. Rev. Lett. 98, 190503 (2007).

10. Riedmatten, H. et al. Long distance quantum teleportation in a quantum relay configuration. Phys. Rev. Lett. 92, 047904 (2004).

11. Ou, Z. Y. \& Lu, Y. J. Cavity enhanced spontaneous parametric downconversion for the prolongation of correlation time between conjugate photons. Phys. Rev. Lett. 83, 2556 (1999).

12. Kuklewicz, C. E., Wong, F. N. C. \& Shapiro, J. H. Time-bin-modulated biphotons from cavity-enhanced down-conversion. Phys. Rev. Lett. 97, 223601 (2006).

13. Bao, X.-H. et al. Generation of narrow-band polarization-entangled photon pairs for atomic quantum memories. Phys. Rev. Lett. 101, 190501 (2008).

14. Fekete, J., Rielander, D., Cristiani, M. \& de Riedmatten, H. Ultranarrow-band photon-pair source compatible with solid state quantum memories and telecommunication networks. Phys. Rev. Lett. 110, 220502 (2013).

15. Harris, S. E. Electromagnetically induced transparency. Phys. Today 50, 36-42 (1997).

16. Du, S., Kolchin, P., Belthangady, C., Yin, G. Y. \& Harris, S. E. Subnatural linewidth biphotons with controllable temporal length. Phys. Rev. Lett. 100, 183603 (2008)

17. Zhao, L. et al. Photon pairs with coherence time exceeding one microsecond. Optica 1, 84-88 (2014).

18. Han, Z., Qian, P., Zhou, L., Chen, J. F. \& Zhang, W. Coherence time limit of the biphotons generated in a dense cold atom cloud. Sci. Rep. 5, 9126 (2015).
19. Zhao, L. et al. Shaping the biphoton temporal waveform with spatial light modulation. Phys. Rev. Lett. 115, 193601 (2015).

20. Thompson, J. K., Simon, J., Loh, H. \& Vuletic, V. A high-brightness source of narrowband, identical-photon pairs. Science 313, 74-77 (2006).

21. Zhang, S. et al. A dark-line two-dimensional magneto-optical trap of ${ }^{85} \mathrm{Rb}$ atoms with high optical depth. Rev. Sci. Instrum. 83, 073102 (2012).

22. Eisaman, M. D. et al. Electromagnetically induced transparency with tunable single-photon pulses. Nature 438, 837-841 (2005).

23. Chen, Q.-F., Shi, B.-S., Feng, M., Zhang, Y.-S. \& Guo, G.-C. Non-degenerate nonclassical photon pairs in a hot atomic ensemble. Opt. Express 16, 21708-21713 (2008).

24. Ding, D.-S., Zhou, Z.-Y., Shi, B.-S., Zou, X.-B. \& Guo, G.-C. Generation of nonclassical correlated photon pairs via a ladder-type atomic configuration: theory and experiment. Opt. Express 20, 11433-11444 (2012).

25. Willis, R. T., Becerra, F. E., Orozco, L. A. \& Rolston, S. L. Photon statistics and polarization correlations at telecommunications wavelengths from a warm atomic ensemble. Opt. Express 19, 14632-14641 (2011).

26. MacRae, A., Brannan, T., Achal, R. \& Lvovsky, A. I. Tomography of a high-purity narrowband photon from a transient atomic collective excitation. Phys. Rev. Lett. 109, 033601 (2012).

27. Du, S., Wen, J. \& Rubin, M. H. Narrowband biphoton generation near atomic resonance. J. Opt. Soc. Am. B 25, C98-C108 (2008).

28. Clauser, J. F. Experimental distinction between the quantum and classical field-theoretic predictions for the photoelectric effect. Phys. Rev. D 9, 853-860 (1974).

29. Grangier, P., Roger, G. \& Aspect, A. Experimental evidence for a photon anticorrelation effect on a beam splitter: a new light on single-photon interferences. Europhys. Lett. 1, 173-179 (1986).

30. Zhang, S. et al. Coherent control of single-photon absorption and reemission in a two-level atomic ensemble. Phys. Rev. Lett. 109, 263601 (2012).

31. Liu, C. et al. Efficiently loading a single photon into a single-sided Fabry-Perot cavity. Phys. Rev. Lett. 113, 133601 (2014).

32. Reim, K. F. et al. Towards high-speed optical quantum memories. Nat. Photon. 4, 218-221 (2010).

33. Oliveira, R. A. et al. Single-photon superradiance in cold atoms. Phys. Rev. A 90, 023848 (2014).

34. Jen, H. H. Superradiant cascade emissions in an atomic ensemble via four-wave mixing. Ann. Phys. 360, 556 (2015).

\section{Acknowledgements}

We thank K. Zhao at Fudan University and L. Zhao at the Hong Kong University of Science and Technology for helpful discussions. T.K.A.C. and L.Z. acknowledge support from the Undergraduate Research Opportunities Program at the Hong Kong University of Science and Technology. The work was supported by Hong Kong Research Grants Council (project no. 16301214)

\section{Author contributions}

S.D. conceived the idea. C.S. and S.D. designed the experiment. C.S., P.C., T.K.A.C. and L.Z. performed the experiment. C.S. did the theory calculation and analysed the data. S.D., C.S. and Y.X. discussed the feasibility of the experiment. S.D. and M.M.T.L. directed the project. All authors contributed to the final manuscript.

\section{Additional information}

Supplementary Information accompanies this paper at http://www.nature.com/ naturecommunications

Competing financial interests: The authors declare no competing financial interests

Reprints and permission information is available online at http://npg.nature.com/ reprintsandpermissions/

How to cite this article: Shu, C. et al. Subnatural-linewidth biphotons from a Doppler-broadened hot atomic vapour cell. Nat. Commun. 7:12783 doi: $10.1038 /$ ncomms12783 (2016).

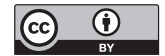

This work is licensed under a Creative Commons Attribution 4.0 International License. The images or other third party material in this article are included in the article's Creative Commons license, unless indicated otherwise in the credit line; if the material is not included under the Creative Commons license, users will need to obtain permission from the license holder to reproduce the material. To view a copy of this license, visit http://creativecommons.org/licenses/by/4.0/

(C) The Author(s) 2016 